\title{
Stable higher-charge discrete vortices in hexagonal optical lattices
}

\author{
Kody J. H. Law and P. G. Kevrekidis \\ Department of Mathematics and Statistics, University of Massachusetts, Amherst, Massachusetts 01003-4515, USA \\ Tristram J. Alexander, Wieslaw Królikowski, and Yuri S. Kivshar \\ Nonlinear Physics Center and Laser Physics Center, Research School of Physical Sciences and Engineering, Australian National \\ University, Canberra, Australian Capital Territory 0200, Australia
}

(Received 7 September 2008; published 3 February 2009)

\begin{abstract}
We show that double-charge discrete optical vortices may be completely stable in hexagonal photonic lattices where single-charge vortices always exhibit dynamical instabilities. Even when unstable the doublecharge vortices typically have a much weaker instability than the single-charge vortices, and thus their breakup occurs at longer propagation distances.
\end{abstract}

DOI: 10.1103/PhysRevA.79.025801

PACS number(s): 42.65.Tg, 05.45.Yv, 42.50.Md

\section{INTRODUCTION}

Some of the most spectacular experiments in the field of nonlinear light propagation in periodic photonic potentials relate to the properties of vortices and vortex flows [1]. Selftrapped phase singularities of optical fields have been observed experimentally in the form of single-charge discrete optical vortices in square photonic lattices [2-4]. In addition, many of the theoretical and experimental studies demonstrated that higher-charge discrete vortices are unstable, similar to the well-studied homogeneous nonlinear systems [1].

In this work, we study single- and double-charge discrete optical vortices in nonsquare periodic photonic lattices [5-11]. In particular, in the framework of the continuous nonlinear model of optically induced lattices generated in saturable nonlinear media, we analyze the existence, stability, and dynamical properties of discrete optical vortices for the case of hexagonal optical lattices. We obtain the somewhat counterintuitive result that double-charge discrete vortices in such lattices appear to be far more robust and structurally stable than single-charge vortices, in agreement with similar findings for Bessel lattices [11]. We verify this finding by demonstrating numerically the generation of a doublecharge vortex with realistic experimental parameters.

It is particularly important to highlight that, although our results will be given with a view toward applications in photorefractive crystals, they are not only relevant to that setting but also directly applicable to two-dimensional hexagonal waveguide arrays (e.g., in glass), showcased in recent experiments (see, e.g., [12] and references therein). Furthermore, they are likely to have direct implications to other areas of physics, such as Bose-Einstein condensates in triangular lattices, the first experiments on which have just been realized [13], or even Debye crystals in dusty plasmas [14]. Another key aspect of the generality of our results is that they should also apply to honeycomb lattices. Hence, the findings presented herein have a bearing on two of the most fundamental nonsquare lattice two-dimensional configurations.

\section{THEORETICAL SETUP}

We study beam propagation through a self-focusing nonlinear medium in the presence of a two-dimensional hexago- nal lattice by employing the continuum model with a saturable nonlinearity. To render our setting completely amenable to the experimentally accessible regime, we use the theoretical model of a photorefractive nonlinear medium, which is known to exhibit strong saturable nonlinearity [8]. Polarization anisotropy of the nonlinear photorefractive response enables one to optically imprint various types of refractive index modulation (optical lattice) which can then be probed by an external beam [15]. Then the propagation of this beam in the presence of an optically induced hexagonal refractive index pattern is governed by the normalized evolution equation

$$
i \frac{\partial u}{\partial z}+D \Delta_{\perp} u-\frac{\gamma u}{1+I_{p}(x, y)+|u|^{2}}=0,
$$

where $u(x, y ; z)$ is the normalized amplitude of the electric field, $z$ is the propagation coordinate, $\Delta_{\perp}$ denotes the transverse Laplacian with respect to $(x, y), D$ is the relevant diffraction coefficient, and $\gamma$ is the material parameter, which is positive or negative depending on whether the nonlinearity is of focusing or defocusing character. The function

$$
\begin{aligned}
I_{p}(x, y)= & I_{g} \mid \exp (i k x)+\exp (-i k x / 2-i k y \sqrt{3} / 2) \\
& +\left.\exp (-i k x / 2+i k y \sqrt{3} / 2)\right|^{2}
\end{aligned}
$$

represents the three-wave interference pattern that induces the hexagonal lattice. The lattice and beam intensities are normalized in units of the dark irradiance of the crystal, $I_{b}$. Throughout this work we use the following experimentally realistic values for the system parameters: $D=z_{s} \lambda /\left(4 \pi n_{0} x_{s}^{2}\right)$ $=18.015$ (for laser wavelength in vacuum $\lambda=532 \mathrm{~nm}$, and average refractive index of the medium $n_{0}=2.35$ ), $\gamma=2.36$, $I_{g}=0.49, k=4 \pi / 3 d$ with a lattice period $d=30 \mu \mathrm{m}$, and where the dimensions $(x, y ; z)$ are in units of $x_{s}=y_{s}=1 \mu \mathrm{m}$ and $z_{s}=1 \mathrm{~mm}$, respectively (see Ref. [7] for further details).

We look for stationary solutions in the form $u(x, y ; z)$ $=U(x, y) \exp (i \beta z) \exp (i m \phi)$, where $U$ is real, $\beta$ is the propagation constant, $\phi$ is the vortex phase, and $m$ is the vortex charge. We solve the resulting nonlinear equation numerically, and the major results are summarized in what follows. 


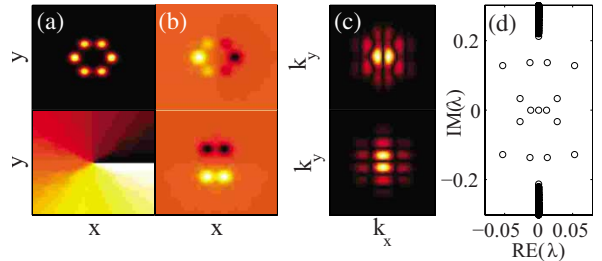

FIG. 1. (Color online) Example of an always unstable singlecharge discrete optical vortex for $\beta=-0.76$ (marked by a circle in Fig. 2). (a) Intensity (top) and phase (bottom); (b) real (top) and imaginary (bottom) components; (c) absolute value of the corresponding Fourier transforms; (d) spectrum of the linearized equation displaying the linear instability of the configuration due to the presence of positive real parts in the eigenvalues $\lambda$ in the spectrum.

\section{NUMERICAL RESULTS}

We begin by considering the simplest six-site vortex structure, that of a single-charge $(m=1)$ discrete vortex. Figure 1 illustrates a typical example, while Fig. 2 shows the single-charge vortex linear stability (top) and power (bottom) as a function of the propagation constant in the semi-infinite band gap of the periodic potential. A positive real part of an eigenvalue in the linear stability spectrum leads to exponential growth of the corresponding linear excitation mode, and therefore to instability of the vortex. Somewhat surprisingly we can see in Fig. 2 (top) that the single-charge vortex has an eigenvalue with a positive real part across its entire region of existence, and therefore the single-charge vortex is always unstable.

In contrast we find that double-charge vortices may be stable [see Fig. 3(a)], and even where unstable the instability is weaker than in the single-charge case (see Fig. 4). In fact, as we can see in Fig. 4 (top), the double-charge vortex has a wide parametric interval where it is completely stable (from $-0.92<\beta<-0.65)$, while outside this range it is unstable due to weak oscillatory instabilities [complex unstable eigenvalues, as evidenced by the spectrum in Fig. 3(b)]. We note that neither the single- nor the double-charge discrete vortex families degenerate into a linear Bloch mode, as one can observe from the saddle-node bifurcation that occurs close to the edge of the first band of the linear spectrum in both Fig. 2 and Fig. 4. The various unstable single- and double-charge vortices which occur along the upper dashed branch in each

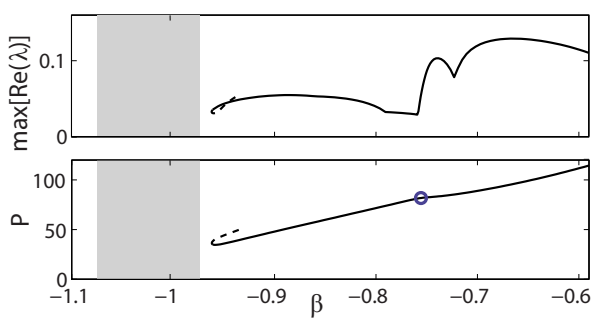

FIG. 2. (Color online) Family of single-charge vortices vs propagation constant $\beta$. Top: maximum real part of the linear stability spectrum. Bottom: power $P=\int_{\infty}^{\infty} U^{2} d x d y$. The circle corresponds to the discrete vortex given in Fig. 1. The dashed line indicates another unstable branch which, for larger $\beta$, bifurcates into different configurations.
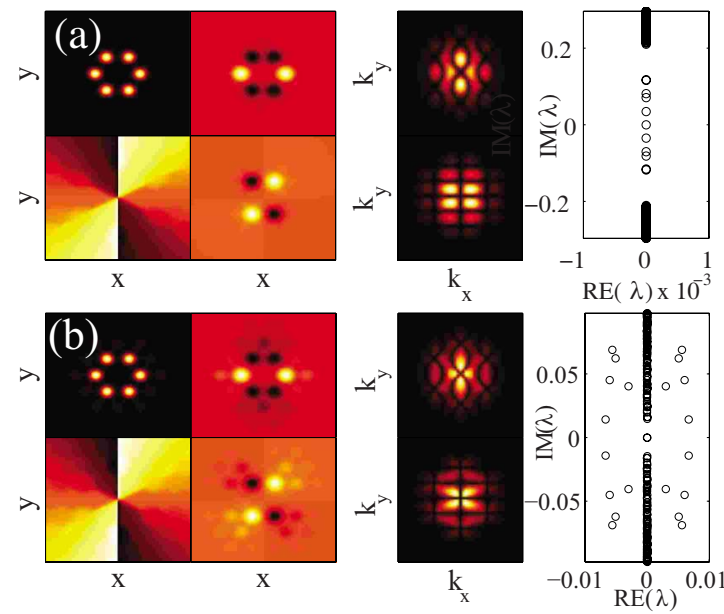

FIG. 3. (Color online) Examples of (a) stable and (b) unstable double-charge discrete optical vortices for $\beta=-0.76$ and -0.96 , respectively (marked, respectively, by the circle and square in Fig. 4). The layout of the panels is the same as in Fig. 1.

figure are not discussed here. The typical evolution of the stable and unstable vortices is illustrated in Figs. 5(a) and 5(b). Even though the single-charge vortex is lower in power than the double-charge vortex, breakup of the former into single-site fundamental discrete solitons occurs around $z$ $=50$, while the double-charge vortex has been propagated to $z=1000$ with no sign of instability.

To further our theoretical understanding, we employ a discrete model. In the latter the analytically tractable anticontinuum limit can be used, for which discrete vortex solutions can be explicitly constructed and a detailed stability analysis can be performed, as has been done for square lattices [16]. In such a setting we consider the six-site configuration with topological charge $m$ over the contour, which takes the form $u_{j}=\exp \left(i \phi_{j}\right) \exp (i z)$, where $\phi_{j}=2 \pi j m / 6$ and $j=1, \ldots, 6$ for the six sites constituting the relevant contour. It is straightforward to see that this configuration yields nontrivial phase profiles for $m=1$ and 2. For these structures, according to the framework of [16], the fundamental vortex will be unstable due to two double real eigenvalue pairs and a single real eigenvalue pair whereas the $m=2$ configuration may be stable. These general results may also be physically under-

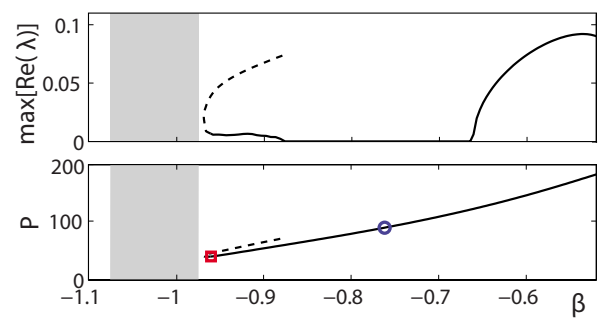

FIG. 4. (Color online) Family of double-charge vortices vs propagation constant $\beta$. Top: maximum real part of linear stability spectrum (when nonzero, this denotes instability). Bottom: power $P=\int_{\infty}^{\infty} U^{2} d x d y$. The circle and square correspond to the stable and unstable discrete vortex configurations shown in Figs. 3(a) and 3(b), respectively. The dashed line indicates an unstable branch which, for larger $\beta$, bifurcates into different configurations. 

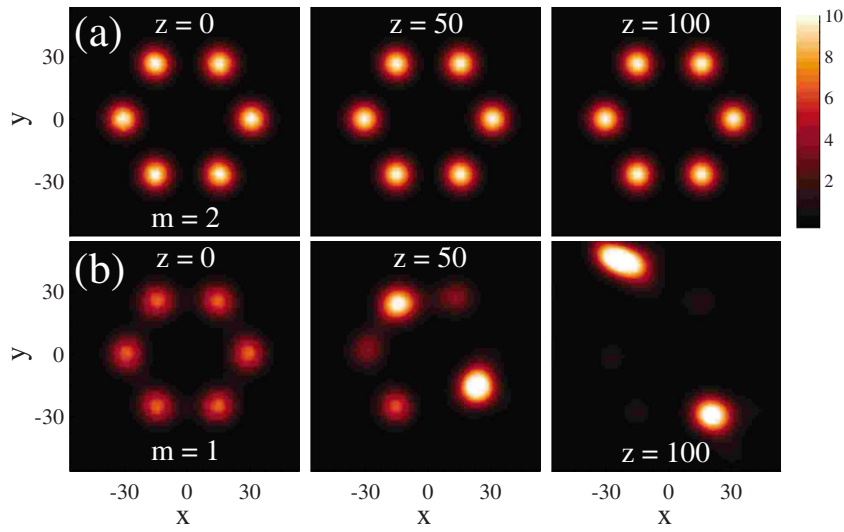

FIG. 5. (Color online) Top three panels (a) depict the evolution of a stable double-charge vortex configuration after a random perturbation with amplitude 5\% of the initial amplitude. Bottom three panels (b) show the evolution of a single-charge vortex configuration. In both cases $\beta=-0.7$. The color bar on the right provides a scale of the intensity [note that the intensities of the single-charge vortex are lower relative to (a) initially and saturated on this scale after breakup].

stood as a consequence of the one-dimensional (1D) modulational instability (MI) results [17] along the 1D (with periodic boundary conditions) six-site contour of the vortex. Such MI considerations predict that configurations where adjacent sites have less than a $\pi / 2$ phase difference (i.e., a single-charge vortex) will be unstable, while those with more than a $\pi / 2$ phase difference (the double-charge case) will be stable.

It is important to point out here that, as the above discrete $1 \mathrm{D}$ contour analysis suggests, our results can qualitatively be extended to other cases where there exists a six-site closed contour, as, e.g., in the so-called honeycomb lattice in which each index maximum has three neighboring maxima instead of six. A typical example of a stable double-charge vortex in a honeycomb lattice is presented in Fig. 6. Furthermore, by extending our consideration of the 1D six-site contour to the case of a defocusing nonlinearity, one can apply a so-called staggering transformation along the contour, $U_{j}=u_{j}(-1)^{j}$. Substitution of this expression in the discrete equation transforms the model from defocusing to focusing (and vice versa). This amounts to translating the phase of every other node along the contour by $\pi$ and, hence, transforming an $m=1(m=2)$ focusing vortex to an $m=2(m=1)$ defocusing vortex, respectively, suggesting a corresponding stability exchange.
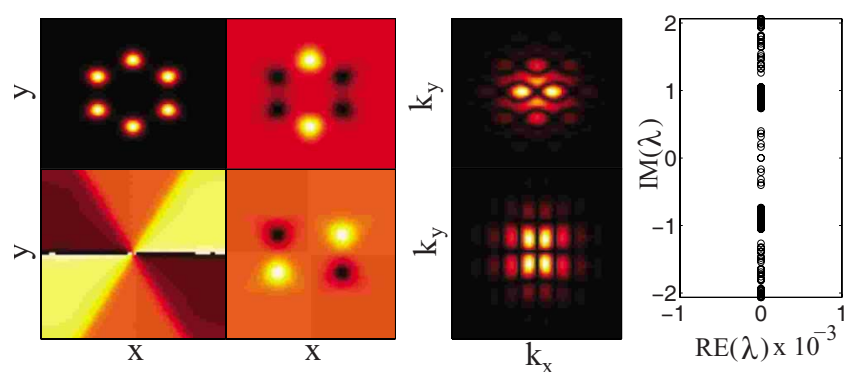

FIG. 6. (Color online) Same set of panels as in Fig. 1 except for a stable double-charge vortex in a honeycomb lattice.

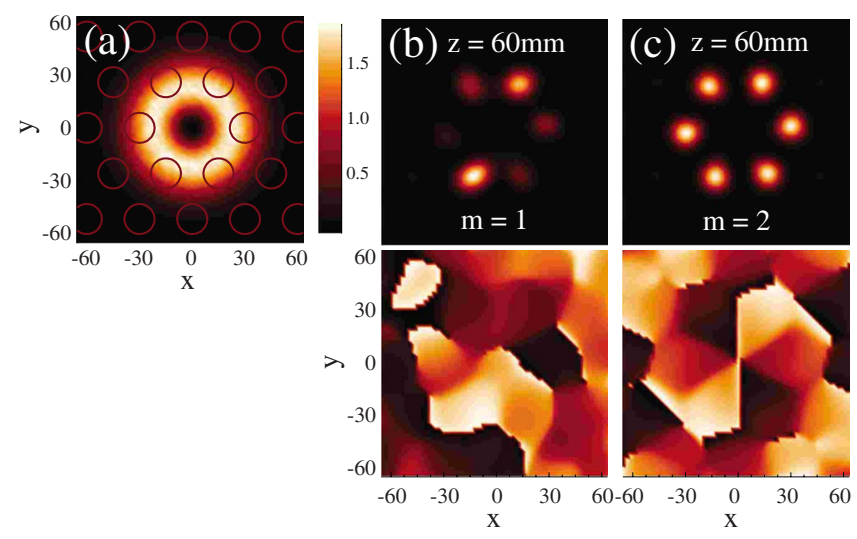

FIG. 7. (Color online) (a) Input beam intensity profile relative to the lattice (position of lattice intensity maxima are shown as rings). The intensity is given by the color bar on the immediate right. Appearance of the beam at $z=60 \mathrm{~mm}$ with different initial vortex phases (intensity not to scale of color bar): (b) single-charge vortex, (c) double-charge vortex. Top panels: intensities; bottom panels: phase.

\section{EXPERIMENTAL PROPOSAL}

Finally, we consider the generation of double-charge vortices and suggest parameters for their experimental observation. For our particular lattice parameters we find that generation of stable double-charge vortices is possible over a wide range of input beam intensities and profiles, at least within our isotropic medium approximation. We consider a Laguerre-Gaussian input beam with the profile shown in Fig. 7 , kept as constant as possible as the input phase is changed, with maximum intensity $\sim 1.8 I_{b}$. In the subsequent evolution we see breakup of the beam into single-site discrete solitons if the initial phase corresponds to a single-charge vortex [Fig. 7(b)], while with an initial double-charge vortex phase we see stable generation of the discrete double-charge vortex [Fig. 7(c)]. Output at $z=60 \mathrm{~mm}$ is shown; however, we have seen no sign of instability in the generated double-charge vortex at a distance of $z=500 \mathrm{~mm}$.

Based on the above considerations, we believe that inputs of the type associated with $m=2$ should be sustained during propagation not only by hexagonal crystals in photorefractive media, but also by two-dimensional hexagonal waveguide arrays (e.g., in glass), showcased in recent experiments [12]. Importantly also, similar results are theoretically expected and have been numerically confirmed (data not shown here) to be valid in the case of honeycomb lattices in such media.

\section{CONCLUSIONS AND OUTLOOK}

We have studied the existence, stability, dynamics, and generation of single- and double-charge discrete optical vortices in two-dimensional hexagonal optical lattices in the framework of a continuum nonlinear model for photorefractive nonlinearity. We have found that, in contrast to square lattices, double-charge vortices can be stable, while single- 
charge vortices are always unstable. Our main finding constitutes a general result for both hexagonal and honeycomb lattices that we expect to be verified experimentally.

There are numerous directions along which it would be interesting to continue the present study. For example, it would be relevant to extend our analysis to the defocusing case for which our discrete theory predicts that the results should be inverted (i.e., that the $m=1$ case should be potentially stable, while the $m=2$ will be unstable). Another direction of interest would be to attempt to generalize such studies to genuinely three-dimensional, nonsquare lattice settings (e.g., in fcc, bcc, or hcp crystals) and observe how threedimensional excitations may behave in these classes of mod- els. Some of these directions are under present consideration and will be reported in future presentations.

\section{ACKNOWLEDGMENTS}

P.G.K. thanks the Nonlinear Physics Center and the Laser Physics Center at the Australian National University for the warm hospitality during his visit to Canberra. P.G.K. also gratefully acknowledges support from NSF Grants No. DMS-0349023, No. DMS-0505663, No. DMS-0619492, and No. DMS-0806762, as well as from the Alexander von Humboldt Foundation. This work has been supported by the Australian Research Council through the Discovery Project scheme.
[1] A. S. Desyatnikov, Yu. S. Kivshar, and L. Torner, in Progress in Optics, edited by E. Wolf (Elsevier, Amsterdam, 2005), Vol. 47, pp. 291-391, and references therein.

[2] D. N. Neshev, T. J. Alexander, E. A. Ostrovskaya, Yu. S. Kivshar, H. Martin, I. Makasyuk, and Z. Chen, Phys. Rev. Lett. 92, 123903 (2004).

[3] J. W. Fleischer, G. Bartal, O. Cohen, O. Manela, M. Segev, J. Hudock, and D. N. Christodoulides, Phys. Rev. Lett. 92, 123904 (2004).

[4] G. Bartal, O. Manela, O. Cohen, J. W. Fleischer, and M. Segev, Phys. Rev. Lett. 95, 053904 (2005).

[5] P. G. Kevrekidis, B. A. Malomed, and Yu. B. Gaididei, Phys. Rev. E 66, 016609 (2002).

[6] V. Koukouloyannis and R. S. MacKay, J. Phys. A 38, 1021 (2005).

[7] O. Peleg, G. Bartal, B. Freedman, O. Manela, M. Segev, and D. N. Christodoulides, Phys. Rev. Lett. 98, 103901 (2007).

[8] C. R. Rosberg, D. N. Neshev, A. A. Sukhorukov, W. Krolikowski, and Yu. S. Kivshar, Opt. Lett. 32, 397 (2007).

[9] T. J. Alexander, A. S. Desyatnikov, and Yu. S. Kivshar, Opt. Lett. 32, 1293 (2007).
[10] B. Terhalle, T. Richter, A. S. Desyatnikov, D. N. Neshev, W. Krolikowski, F. Kaiser, C. Denz, and Yu. S. Kivshar, Phys. Rev. Lett. 101, 013903 (2008).

[11] Y. V. Kartashov, A. Ferrando, A. A. Egorov, and L. Torner, Phys. Rev. Lett. 95, 123902 (2005).

[12] A. Szameit, Y. V. Kartashov, F. Dreisow, M. Heinrich, V. A. Vysloukh, T. Pertsch, S. Nolte, A. Tünnermann, F. Lederer, and L. Torner, Opt. Lett. 33, 663 (2008).

[13] See, e.g., C. Becker, P. Soltan-Panahi, J. Kronjäger, S. Stellmer, K. Bongs, and K. Sengstock, in CLEO/Europe and IQEC 2007 Conference Digest, (Optical Society of America, Washington, DC, 2007), Paper. IB1_1.

[14] V. Koukouloyannis and I. Kourakis, Phys. Rev. E 76, 016402 (2007).

[15] N. K. Efremidis, S. Sears, D. N. Christodoulides, J. W. Fleischer, and M. Segev, Phys. Rev. E 66, 046602 (2002).

[16] D. E. Pelinovsky, P. G. Kevrekidis, and D. J. Frantzeskakis, Physica D 212, 20 (2005).

[17] Yu. S. Kivshar and M. Peyrard, Phys. Rev. A 46, 3198 (1992); D. N. Christodoulides and R. I. Joseph, Opt. Lett. 13, 794 (1988). 